participants were assessed for PTSD, depression, anxiety and alcohol misuse; OI; performed neurocognitive measures of dorsolateral prefrontal, lateral prefrontal and mesial temporal functioning; and completed a self-report assessment of aggression.

Results: War veterans with PTSD exhibited significant OI deficits (OID) compared with controls, despite uncompromised performance on tests of verbal fluency, verbal paired associate learning, visuospatial planning and construction, and attention and motor inhibition. OIDs remained after covaring for IQ, anxiety, depression and alcohol misuse. No significant smoking or medication effects were observed. OIDs were significant predictors of aggressive and impulsive behaviour.

Conclusions: This research contributes to emerging evidence of orbitoprefrontal dysfunction in the pathophysiology underlying PTSD. This is the first study to report OID as a strong predictor of impulsive and aggressive behaviour in this clinical population. It prompts research to further explore the potential diagnostic utility of OIDs in the assessment of PTSD. Such measures may help delineate the complexity of PTSD symptom presentation and support the targeted interventions for impulsive aggressive behaviour.

\section{Converging evidence from taxometric analyses confirms a cognitive subtype of schizophrenia with distinct genetic basis}

\section{Dragovic, A Jablensky}

Centre for Clinical Research in Neuropsychiatry, The University of Western Australia

Background: Two distinct schizophrenia subtypes have recently been identified by the Western Australian Family Study of Schizophrenia by grade of membership (GoM) analysis of the phenotype (Hallmayer et al. 2005; Jablensky 2006): one characterized by pervasive cognitive deficit (CD) and low scores on personality trait measures and one featuring significant personality deviations but with relatively intact cognitive performance [cognitively spared (CS)]. Whole-genome scan of 93 families discovered significant linkage to 6p25-22 for the CD subtype, while the linkage for CS subtype was definitively excluded for that region. The aim of this study was to investigate by another method whether differences between these subtypes are qualitative or quantitative.

Methods: Several taxometric procedures, originally proposed by P. Meehl (1994, 1996, 1998), were used to analyze taxonicity of schizophrenia subtypes: meanabove-mean-below-a-cut, maximum-eigenvalue and latent mode analyses in a sample of 138 individuals with schizophrenia and schizophrenia spectrum disorders.

Results: Three independent taxometric procedures showed consistently a latent taxonomic structure in our sample of patients with schizophrenia. Estimated mean base rates for $\mathrm{CD}$ taxon ranged from 0.37 to 0.43 , suggesting that about $40 \%$ of patients with schizophrenia belong to this taxon.

Conclusions: CD schizophrenia subtype is discrete, that is, taxonic. Taxometric analyses have further corroborated the existence of an etiologically discrete schizophrenia subtype.

\section{Coregulation of genes in the mouse brain following antipsychotic drug treatment}

\section{Duncan', A Chetcuti' ${ }^{2}$ P Schofield ${ }^{3}$ \\ ${ }^{1}$ Garvan Institute of Medical Research; ${ }^{2}$ Neuroscience Institute of Schizophrenia and Allied Disorders (NISAD); and ${ }^{3}$ Prince of Wales Medical Research Institute, Sydney, Australia}

Background: Schizophrenia is a major psychiatric disorder that affects approximately $1 \%$ of people during their lifetimes. Antipsychotic drugs are the most effective treatment for the psychotic phase of schizophrenia, although their mechanism of action remains largely unknown.

Methods: We have treated mice with one of three antipsychotics to create animal models of antipsychotic drug action. Control mice were treated with saline. Drug treatment was performed by means of daily intraperitoneal injections for 1 and 4 weeks. RNA was extracted from the brains of these mice and hybridized to whole-genome microarray chips. Validation of mRNA expression changes in selected genes was undertaken using quantitative polymerase chain reaction (PCR) and protein expression was investigated using Western blot analysis.

Results: Data analysis showed that many genes were dysregulated by antipsychotic drug treatment, including those involved in signal transduction, synaptic transmission and neurogenesis. Genes were selected for further analysis based upon their coregulation by different antipsychotics, chromosomal location or known molecular function. Changes in gene expression were confirmed for 13 of 19 genes thus far analyzed by quantitative PCR. Western blot analysis indicated that these changes in mRNA levels are translated into protein expression changes in at least two genes; neural precursor cell developmentally downregulated gene 4 (Nedd4) and potassium voltagegated channel, shaker-related subfamily, member 1 (Kcnal). 